\title{
Impact of the first national COVID-19 lockdown on management in a gynecological emergency department: illustration of the resilience of a health center
}

\author{
Elisabeth Asselineau', Florence Llouquet ${ }^{1}$, Antoine Bourret ${ }^{1}$, Laetitia Campin'1 , Charles Chapron ${ }^{1,2}$, \\ Dan Chaltiel ${ }^{3}$, Florie Pirot ${ }^{1}$
}

'Assistance Publique - Hôpitaux de Paris (AP-HP), Hôpital Universitaire Paris Centre (HUPC), Centre Hospitalier Universitaire (CHU) Cochin, Department of Gynecology, Obstetrics and Reproductive Medicine, Paris, France

2Université Paris Descartes, Sorbonne Paris Cité, Faculté de Médecine, Paris, France

${ }^{3}$ Department of Biostatistics and Epidemiology, Gustave Roussy, University Paris-Saclay, Villejuif, France

\footnotetext{
Abstract

Introduction: The implementation of the first national lockdown during COVID-19 epidemic has forced primary care to adapt quickly.

Aim: The main objective of this work was to evaluate the effect of the first lockdown imposed on the French population in 2020 on the number of admissions in gynecological emergencies in one center, in comparison with 2019. The secondary objectives were the study of diagnostics, analysis of the treatments and comparison of the complication rates between the two periods studied.

Material and methods: A retrospective study was conducted in the gynecological EDs of Cochin Port Royal Hospital (Paris, France) regarding all admissions from 1 February to 30 April for 2019 and 2020.

Results: A total of 4911 women were admitted to the gynecological EDs. The lockdown was associated with an average decrease of 20.3 patients per day, independently of the year and period. We noted a significant increase in the odds of deferred surgery $(p=0.02)$ and hospitalizations $(p=0.03)$, but this was not significant for immediate emergency surgery $(p=0.4)$. Moreover, there has been a significant increase in the odds of a new consultation with complication $(p<0.001)$.

Conclusions: The COVID-19 lockdown reduced the admission rate to gynecological EDs in our center, but continues to test the capacity of health systems to manage emergencies.

Key words: COVID-19, gynecological emergency department, lockdown.
} Centre Hospitalier Universitaire (CHU) Cochin, Department of Gynecology, Obstetrics and Reproductive Medicine, Paris, France, e-mail: florie.pirot@gmail.com 


\section{Introduction}

The healthcare organization in France is based on liberal medicine and hospitals (mostly public), and the strongest link between them is the emergency department. In 2018, there were 636 French health establishments with emergency room authorization (1 per 10000 inhabitants). Of these, $76 \%$ were public sector facilities. The number of emergency room visits has steadily increased since the 1990s: 21.1 million emergency room visits were recorded in metropolitan France in 2018, which is $2 \%$ more than in 2017 , and $30 \%$ more than ten years ago [1]. In 2014, this represented a cost of 2.5 million euros borne by the national health insurance system [2].

To the best of our knowledge, few studies have studied the attendance rates and reasons for visiting gynecological emergencies [3, 4]. A 2012 study showed that only 12\% of patients presenting to the emergency department were hospitalized and $4.5 \%$ were operated on [5]. The authors concluded that the majority of patients had been consulted for minor pregnancy pathologies or gynecological pathologies that are not emergencies and that could have been treated in a scheduled consultation, reaffirming the preponderant role of the general practitioners or liberal gynecologists (primary care).

In December 2019, a new epidemic disease, the coronavirus disease-19 (COVID-19), was found in Hubei province of China. The presence of COVID-19 was manifested by several symptoms, ranging from asymptomatic/mild symptoms to severe illness and death. The reproduction rate of COVID-19 proved to be very high, and the infection has spread rapidly around the world. In this context, the WHO announced a public health emergency of international concern.

The virus was confirmed to have reached France on 24 January 2020, when the first COVID-19 case in both Europe and France was identified in Bordeaux [6]. After a few days, the virus spread throughout the country as it did in neighboring European countries. The government issued a series of restrictive measures to limit the contagion spread. The 12 March 2020 decree defined the beginning of the socalled "lockdown" phase, which led to the closure of all commercial and educational activities as well as all restaurants, prohibiting the gathering of people in public places. The lockdown continued until 11 May 2020 [7].

The implementation of these major measures was associated with a complete reorganization of healthcare centers with prioritization of emergencies, postponement of elective surgery, and development of teleconsultations. The impact of lockdown on the incidence of medical-surgical emergencies is still being discussed. Some researchers have predicted by modeling an increase in late-diagnosed cancers [8]. Rahul et al. noted an increase in the number of emergencies (here neurosurgery) but a $40 \%$ decrease in the number of procedures performed, illustrating a change in the pattern of surgical emergencies [9].

To the best of our knowledge, only one study has been published in the initial phase of lockdown on emergency room attendance during this period of pandemic and population containment [10]. Authors described a significant decrease in consultations for all pathologies, except for those characterized by surgical emergencies or immediate vital emergencies, suggesting a better use of the emergency department by patients. Nonetheless, other more recent studies now contradict it by emphasizing the increase in morbidity and mortality related to under-diagnosis $[11,12]$.

Because good healthcare organization and coordination are essential and crucial in times of crisis, it seemed very important to assess the impact of lockdown on French gynecological emergencies to improve departments' organization, should there be another similar crisis in the future.

\section{Aim}

Therefore, the main objective of this work was to evaluate the impact of the first COVID-19 lockdown imposed on the population in 2020 on the number of admissions in gynecological emergencies in the maternity unit of Cochin Port-Royal Hospital (Paris France), in comparison with 2019.

The secondary objectives were the study of diagnostics, analysis of the proposed treatments and comparison of the complication rate leading to a new unscheduled consultation between 2019 and 2020 .

\section{Material and methods}

\section{Population and periods}

This retrospective observational study was conducted in the Emergency Department of Obstetrics and Gynecology of the Cochin Port Royal University hospital (Paris, France). All admissions to the gynecological emergency department (ED) between 1 February and 30 April for 2019 and 2020 were included in the study. Admissions during the second and third trimesters of pregnancy and the puerperium were excluded because they were part of the non-deferrable hospital surveillance and were the subject of a specific dedicated organization.

In order to study the first lockdown effect, we split both 2019 and 2020 into two periods separated by the lockdown calendar date: P1 from 1 February to $15 \mathrm{March}$, and P2 from 16 March to 30 April. Patients from 2019 were included to account for a seasonal effect that could have explained the difference between the time after and before lockdown. Comparisons must be made by checking whether the evolution between periods 1 and 2 is different in 2019 and 2020 .

\section{Clinical characteristics}

Characteristics of patients were acquired from computerized medical files. There were no missing values in the features that were studied.

For each patient included, we collected demographic information (age, obesity status, smoking status), vital signs on admission, pain evaluation according to the visual analog scale (0 to 10/10), reason for consultation (pelvic pain, bleeding during $1^{\text {st }}$ trimester of pregnancy, bleeding without pregnancy, follow-up, and other), final diagnosis, proposed management (hospitalization, emergency surgery, deferred surgery (i.e. semi-emergency), ambulatory treatment, and 
ambulatory examinations), proposed follow-up (reconvening/recovery, follow-up teleconsultation, or none), and outcomes (new consultation, with or without complications).

For the analysis, we divided the diagnoses into 4 groups:

- Vital emergencies: ruptured ectopic pregnancy, complicated spontaneous miscarriage (pain and/or hemorrhage), complicated pelvic inflammatory disease (requiring antibiotic infusion or surgery).

- Relative emergencies: non-ruptured ectopic pregnancy, non-complicated pelvic inflammatory disease, ovarian cyst (without ovary torsion), pelvic wound, ovarian hyperstimulation syndrome, cancer suspicion, intra-abdominal collection, intra-uterine trophoblastic retention.

- Non-emergency pathology: intrauterine pregnancy, pregnancy of unknown location, aborted pregnancy, hydatidiform mole, hyperemesis gravidarum, functional pathology (hormonal bleed or pain), endometriosis and adenomyosis, uterine myoma, polyp, pelvic organ prolapse, vulvar pathology, breast pathology, skin or scar pathology, foreign object.

- Non-gynecological pathology: cystitis and acute pyelonephritis, sexual abuse, non-gynecological etiology. In addition, we have also classified the complications that led to a new consultation by degree of urgency:

- None (for example patients who came because they did not find a laboratory or ultrasound center).

- Minor: anemia or disturbance of the bio-balance, scarring complications, trophoblastic retention.

- Moderate: aggravation of infection, surgical site infection, pelvic collection (hematoma).

- Severe: complicated miscarriage, ruptured ectopic pregnancy, ovary torsion, new surgery.

\section{Statistical analysis}

For the primary objective, a linear model was prepared to explain the number of admissions per day regarding year (2019 or 2020), period (period 1 or period 2), and between these two variables. The interaction term therefore represents the effect of the lockdown, independent of the year and period effect.

For the secondary objectives, variables were studied using logistic models with the same terms. Since there was no reference level to describe our multilevel variables, multinomial logistic models could not be used. Therefore, multiple standard logistic models were used instead considering each level as an independent variable. The slight misestimation of coefficients was considered neglectable.

Since there was no formal hypothesis to explain the behavior of the population during lockdown, no further adjustment could be considered.

No ethical approval was requested from the different Institutional Review Boards for a simple review of the medical records, since the collection of these data was performed during clinical practice.

All analyses were performed using R (version 4.0.1) and the "tidyverse" packages $[13,14]$ with a significance level of $5 \%$ for 2 -sided tests.

\section{Results}

\section{Population}

A total of 4911 women were admitted to the gynecologic emergency department of Cochin Port-Royal Hospital (Paris, France) and have been evaluated in this study. There were 2902 women in 2019 (1377 in period 1 and 1525 in period 2) and 2009 in 2020 (1393 in period 1 and 616 in period 2).

Basic characteristics of included women are reported for each period in Table I.

A graphic representation of the admission rate to the emergency department of our center during the study's timeframe is given in Figure 1. This rate decreased dramatically after the date of lockdown in 2020 while it did not in 2019.

The result of the primary analysis is described in Table II. In the linear model, the lockdown was associated with an average decrease of 20.3 patients per day, independently of the year and period.

\section{Diagnoses, treatment and issue}

The results of the secondary analyses are described in Table III. In the logistic models, there was no significant association between the lockdown and other periods on the division of the different diagnoses, with a slight trend to decrease of the odds for non-gynecological pathologies $(p=0.06)$.

However, lockdown was associated with the outcome as there was a significant decrease in the odds of not coming again to the EDs $(p<0.001)$ and a significant increase in the odds of a new consultation with complication $(p<0.001)$.

A sensitivity analysis was performed without considering trophoblastic retention and complicated miscarriage as complications and the association remained significant to measure the impact of aborted pregnancies $(p=0.02)$.

In order to investigate this increase in the advent of complications, we used 3 more logistic models showing a significant increase in the odds of obesity $(p=0.009)$ and of having an aborted pregnancy $(p=0.01)$, but no statistical difference in methotrexate use in ectopic pregnancy $(p=$ 0.7). These variables are described in Table IV.

Lockdown was associated with changes in the follow-up as there was a significant decrease in the odds of reconvening $(p<0.001)$ and a significant increase in the odds of teleconsultation $(p<0.001)$.

Finally, regarding the management of women in EDs, we noted a significant increase in the odds of deferred surgery $(p=0.02)$ and hospitalizations $(p=0.03)$, but this was not significant for immediate emergency surgery $(p=0.4)$.

\section{Discussion}

In this study, the number of admissions to gynecological emergencies decreased significantly during the lockdown period, regardless of the period of the year. This significant decrease in gynecological ED visits during the period of the COVID-19 epidemic could be explained by the implementation of restrictive measures and fear of contamination, as suggested by some authors $[15,16]$. But actually, this result 
Table I. Description of the population in both 2019 and 2020 and in both periods. Period 1 goes from 1 February to 15 March, and Period 2 from 16 March to 30 April. Port-Royal Maternity, $N=4911$ admissions to the $\mathrm{ED}^{\mathrm{a}}$

\begin{tabular}{|c|c|c|c|c|c|}
\hline \multirow[t]{2}{*}{ Variable } & \multirow[t]{2}{*}{ Total } & \multicolumn{2}{|c|}{2019} & \multicolumn{2}{|c|}{2020} \\
\hline & & Period 1 & Period 2 & Period 1 & Period 2 \\
\hline Age & $33.9(9.2)$ & $33.5(9.6)$ & $34.0(9.0)$ & $33.6(8.9)$ & $35.4(9.3)$ \\
\hline Heart rate & $84.5(14.6)$ & $84.1(14.6)$ & $83.8(14.1)$ & $84.6(14.7)$ & $86.8(15.6)$ \\
\hline Temperature & $36.8(0.5)$ & $36.8(0.5)$ & $36.8(0.4)$ & $36.8(0.5)$ & $36.7(0.5)$ \\
\hline Systolic blood pressure & $12.3(1.6)$ & $12.4(1.5)$ & $12.4(1.7)$ & $12.2(1.6)$ & $12.3(1.6)$ \\
\hline Diastolic blood pressure & $7.6(1.2)$ & $7.6(1.2)$ & $7.6(1.2)$ & $7.5(1.2)$ & $7.6(1.1)$ \\
\hline Subjective Pain Scale & $3.0(3.1)$ & $3.3(3.1)$ & $3.0(3.2)$ & $2.8(3.0)$ & $2.6(3.0)$ \\
\hline Smoking & $454(9 \%)$ & $113(8 \%)$ & $132(9 \%)$ & $159(11 \%)$ & $50(8 \%)$ \\
\hline Obesity & $297(6 \%)$ & $65(5 \%)$ & $80(5 \%)$ & $81(6 \%)$ & $71(12 \%)$ \\
\hline Referred patient & $603(12 \%)$ & $187(14 \%)$ & $157(10 \%)$ & $166(12 \%)$ & $93(15 \%)$ \\
\hline Prescribed blood test & $878(18 \%)$ & $177(13 \%)$ & $98(6 \%)$ & $393(28 \%)$ & $210(34 \%)$ \\
\hline Office surgery & $226(5 \%)$ & $67(5 \%)$ & $65(4 \%)$ & $63(5 \%)$ & $31(5 \%)$ \\
\hline Hospitalization & $382(8 \%)$ & $109(8 \%)$ & $116(8 \%)$ & $95(7 \%)$ & $62(10 \%)$ \\
\hline Scheduling a surgery & $202(4 \%)$ & $57(4 \%)$ & $45(3 \%)$ & $62(4 \%)$ & $38(6 \%)$ \\
\hline \multicolumn{6}{|l|}{ Proposed follow-up: } \\
\hline None & $3771(77 \%)$ & $1098(80 \%)$ & $1154(76 \%)$ & $1053(76 \%)$ & $466(76 \%)$ \\
\hline Reconvening & $1092(22 \%)$ & $266(19 \%)$ & $368(24 \%)$ & $338(24 \%)$ & $120(19 \%)$ \\
\hline Teleconsultation & $48(1 \%)$ & $13(1 \%)$ & $3(0.2 \%)$ & $2(0.1 \%)$ & $30(5 \%)$ \\
\hline \multicolumn{6}{|l|}{ Outcome: } \\
\hline New consultation with complication & $188(4 \%)$ & $42(3 \%)$ & $24(2 \%)$ & $65(5 \%)$ & $57(9 \%)$ \\
\hline New consultation without complication & $356(7 \%)$ & $112(8 \%)$ & $116(8 \%)$ & $88(6 \%)$ & $40(6 \%)$ \\
\hline No more consultation & $4367(89 \%)$ & $1223(89 \%)$ & $1385(91 \%)$ & $1240(89 \%)$ & $519(84 \%)$ \\
\hline \multicolumn{6}{|l|}{ Final diagnosis: } \\
\hline Non-emergency pathology & $3510(71 \%)$ & $1005(73 \%)$ & $1091(72 \%)$ & $972(70 \%)$ & $442(72 \%)$ \\
\hline Relative emergencies & $776(16 \%)$ & $205(15 \%)$ & $244(16 \%)$ & $222(16 \%)$ & $105(17 \%)$ \\
\hline Non-gynecological pathology & $487(10 \%)$ & $132(10 \%)$ & $154(10 \%)$ & $152(11 \%)$ & $49(8 \%)$ \\
\hline Vital emergencies & $138(3 \%)$ & $35(3 \%)$ & $36(2 \%)$ & $47(3 \%)$ & $20(3 \%)$ \\
\hline \multicolumn{6}{|l|}{ Indication of consultation: } \\
\hline Pelvic pain & $1495(30 \%)$ & $426(31 \%)$ & $541(35 \%)$ & $357(26 \%)$ & $171(28 \%)$ \\
\hline Follow-up & $977(20 \%)$ & $212(15 \%)$ & $331(22 \%)$ & $310(22 \%)$ & $124(20 \%)$ \\
\hline Bleeding during $1^{\text {st }}$ trimester of pregnancy & $812(17 \%)$ & $257(19 \%)$ & $182(12 \%)$ & $260(19 \%)$ & $113(18 \%)$ \\
\hline Bleeding without pregnancy & $489(10 \%)$ & $142(10 \%)$ & $163(11 \%)$ & $138(10 \%)$ & $46(7 \%)$ \\
\hline Other & $1138(23 \%)$ & $340(25 \%)$ & $308(20 \%)$ & $328(24 \%)$ & $162(26 \%)$ \\
\hline \multicolumn{6}{|l|}{ Ambulatory treatment: } \\
\hline None & $2338(48 \%)$ & 737 (54\%) & $932(61 \%)$ & $464(33 \%)$ & $205(33 \%)$ \\
\hline Only painkillers & $1249(25 \%)$ & $219(16 \%)$ & $252(17 \%)$ & $563(40 \%)$ & $215(35 \%)$ \\
\hline Other & $1324(27 \%)$ & $421(31 \%)$ & $341(22 \%)$ & $366(26 \%)$ & 196 (32\%) \\
\hline \multicolumn{6}{|l|}{ Prescribed radiological exam: } \\
\hline None & $3796(77 \%)$ & $1061(77 \%)$ & $1245(82 \%)$ & $1047(75 \%)$ & 443 (72\%) \\
\hline Radiologic external exam & $994(20 \%)$ & $279(20 \%)$ & $245(16 \%)$ & $303(22 \%)$ & $167(27 \%)$ \\
\hline Other diagnostic exams & $121(2 \%)$ & $37(3 \%)$ & $35(2 \%)$ & $43(3 \%)$ & $6(1 \%)$ \\
\hline
\end{tabular}

aValues are described as "Mean (SD)" for numerical variables and as "Count (percentage)" for categorical variables. 
is multifactorial and the secondary analyses support a few additional leads.

In our study, the lockdown, although having an unquestionable quantitative impact, did not seem to have any qualitative consequences. Indeed, there was no statistical impact of the lockdown on division of the 4 diagnosis groups. It was expected that non-urgent diagnoses would decrease in priority, but our results confirm the misuse of gynecological EDs even in times of health crisis. Indeed, in most Western countries, emergencies are increasingly being used for non-emergency medical care, especially during pregnancy [17]. According to a North American literature review conducted in 2013, nearly one-third of patients seen in emergency departments have "non-urgent" problems that could have been treated in an ambulatory setting [18]. Using emergency departments for non-urgent care can lead to excessive healthcare expenditures

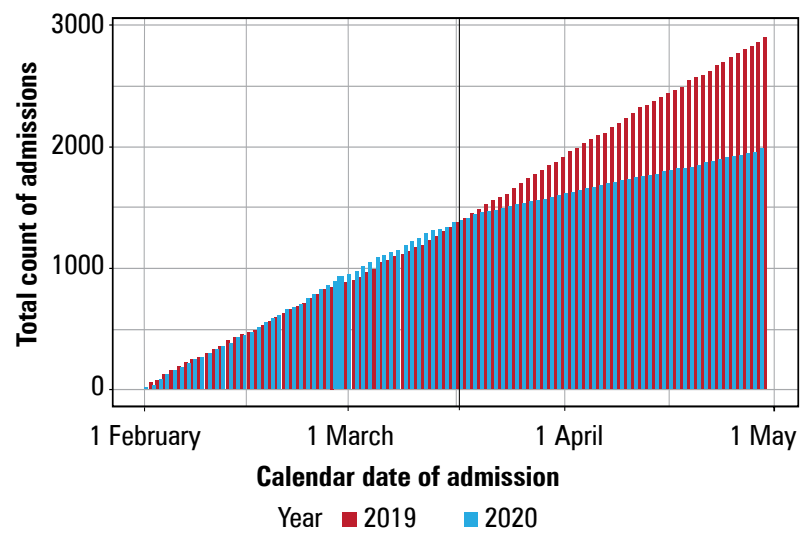

Figure 1. Cumulative sum of admissions to the emergency department of Port-Royal hospital in the period between 1 February and 30 April, in 2019 and in 2020. The vertical line represents the date of the first lockdown in France

Table II. Results of the primary analysis linear model: number of consultations per day as a function of the year (2019 or 2020), the period (period 1 or period 2), and the interaction/relation between these two variables ${ }^{\mathrm{a}}$

\begin{tabular}{|l|c|c|c|}
\hline Term & Coefficient & $95 \%$ Cl & $P_{\text {-value }}^{\text {b }}$ \\
\hline Intercept & 30.6 & {$[28.4 ; 32.8]$} & $<0.001$ \\
\hline Year (ref = 2019) & -0.3 & {$[-3.4 ; 2.8]$} & 0.84 \\
\hline Period (ref = Period 1) & 4.1 & {$[0.9 ; 7.2]$} & 0.01 \\
\hline Interaction of the year and period & -20.3 & {$[-24.8 ;-15.9]$} & $<0.001$ \\
\hline
\end{tabular}

aThe interaction term therefore represents the effect of the lockdown, independent of the year and period effect. ${ }^{\text {b}}$ The $p$-values were computed using Wald tests for coefficient nullity.

Table III. Results of the secondary analysis logistic models: each factor as a function of the year (2019 or 2020), the period (period 1 or period 2), and the interaction between these two variables. Port-Royal Maternity, $N=4911$ admissions to the ED

\begin{tabular}{|c|c|c|c|c|}
\hline Variable & $\mathrm{OR}^{\mathrm{a}}$ & $95 \% \mathrm{Cl}$ & $P$-value ${ }^{c}$ & * \\
\hline \multicolumn{5}{|l|}{ Final diagnosis ${ }^{b}$} \\
\hline Non-emergency pathology & 1.18 & {$[0.9 ; 1.5]$} & 0.22 & \\
\hline Relative emergencies & 1.00 & {$[0.7 ; 1.4]$} & 1.0 & \\
\hline Non-gynecological pathology & 0.67 & {$[0.4 ; 1.0]$} & 0.06 & . \\
\hline Vital emergencies & 1.04 & {$[0.5 ; 2.1]$} & 0.9 & \\
\hline \multicolumn{5}{|l|}{ Outcome $^{b}$} \\
\hline No more consultation & 0.53 & {$[0.4 ; 0.8]$} & $<0.001$ & $* * *$ \\
\hline New consultation without complication & 1.11 & {$[0.7 ; 1.8]$} & 0.67 & \\
\hline New consultation with complication & 4.10 & {$[2.2 ; 7.7]$} & $<0.001$ & $* * *$ \\
\hline \multicolumn{5}{|l|}{ Proposed follow-up ${ }^{b}$} \\
\hline None & 1.27 & {$[1.0 ; 1.7]$} & 0.098 & . \\
\hline Reconvening & 0.57 & {$[0.4 ; 0.8]$} & $<0.001$ & $* * *$ \\
\hline Teleconsultation & 172.16 & {$[31.1 ; 1566.9]$} & $<0.001$ & $* * *$ \\
\hline Hospitalization & 1.60 & {$[1.0 ; 2.5]$} & 0.03 & * \\
\hline Emergency surgery & 1.29 & {$[0.7 ; 2.2]$} & 0.4 & \\
\hline Deferred surgery & 2.00 & {$[1.1 ; 3.6]$} & 0.02 & * \\
\hline Obesity $^{d}$ & 1.9 & {$[1.2 ; 3.0]$} & 0.009 & ** \\
\hline Aborted pregnancy ${ }^{d}$ & 1.8 & {$[1.1 ; 2.9]$} & 0.01 & * \\
\hline Methotrexate treatment in ectopic pregnancies $(N=323)^{\mathrm{d}}$ & 0.8 & {$[0.3 ; 2.3]$} & 0.7 & \\
\hline
\end{tabular}

${ }^{a}$ Odds ratio for the interaction/relation term, which represents the effect of the lockdown, independent of the year and period effect. ${ }^{b}$ For multilevel factors, multiple logistic models were fit on dummy variables. ${ }^{c}$ The $p$-values were computed using Wald tests for coefficient $\left(\log\right.$-OR) nullity. ${ }^{\mathrm{d}}$ Exploratory, unplanned analysis performed to investigate the results of outcome. 
Table IV. Description of supplemental variables

\begin{tabular}{|c|c|c|c|c|c|}
\hline \multirow[t]{2}{*}{ Variable } & \multirow[t]{2}{*}{ Total } & \multicolumn{2}{|c|}{2019} & \multicolumn{2}{|c|}{2020} \\
\hline & & Period 1 & Period 2 & Period 1 & Period 2 \\
\hline \multicolumn{6}{|l|}{ Complication: } \\
\hline None & $4723(96 \%)$ & 1335 (97\%) & 1501 (98\%) & $1328(95 \%)$ & $559(91 \%)$ \\
\hline Minor & $93(2 \%)$ & $20(1 \%)$ & $10(1 \%)$ & $29(2 \%)$ & $34(6 \%)$ \\
\hline Moderate & $19(0.4 \%)$ & $4(0.3 \%)$ & $3(0.2 \%)$ & $6(0.4 \%)$ & $6(1 \%)$ \\
\hline Severe & $76(2 \%)$ & $18(1 \%)$ & $11(1 \%)$ & $30(2 \%)$ & $17(3 \%)$ \\
\hline Aborted pregnancy & $312(6 \%)$ & $70(5 \%)$ & $82(5 \%)$ & $89(6 \%)$ & $71(12 \%)$ \\
\hline Methotrexate treatment in ectopic pregnancies $(N=323)$ & $90(27.86 \%)$ & $18(26.47 \%)$ & $29(26.61 \%)$ & $30(30.61 \%)$ & $13(27.08 \%)$ \\
\hline
\end{tabular}

through unnecessary testing and treatment, and, in this peculiar context, to new infection cases. However, most of the non-urgent consultations over our study period were actually due to the abrupt closure of biological laboratories and external imaging centers. Thus, it is not only a question of reminding the population of the usefulness of gynecological emergencies but also of making these outpatient services available in the event of a new crisis.

We noticed an increase in the number of hospitalizations and deferred surgeries. Thus, despite the reduction in the number of consultations and although the diagnostic profiles were similar, the lockdown did have a significant effect on the management of gynecological ED: deferred surgery in order to optimize the hospital beds, continue to operate emergency cases that required it, and more hospital care when primary care is no longer sufficient. These results are in line with those of Grandi et al., who also suggested that the decrease in admissions to gynecological emergencies came with optimization of care [10]. This change in pattern of emergencies may be due to later consultations (delay in diagnosis) or to the closure of nearby polyclinics. The adaptation of primary care during the COVID-19 epidemic disrupted the usual paradigms of gynecological EDs.

The outcome of the consultations also reflected this adaptation in times of crisis. The decrease in the number of reconvening was inversely proportional to the emergence of teleconsultation. Teleconsultation was also maintained after the health crisis in the department in order to limit non-urgent consultations while maintaining a medical follow-up.

Regarding the outcome of our patients, the increase in the rate of complications can be considered as a collateral damage of this change in management rather than attributable to COVID-19 pandemic. Several explanations could be considered.

First, the crisis caused the closure of local primary care and ultrasound centers, which usually manage minor complications and aborted pregnancies. This might explain the increase of these two conditions during the lockdown period in our data.

Second, mostly patients in bad condition might dare to come in the ED during the lockdown. Indeed, MacLead et al. found a "lockdown effect" in general emergency: patients had significantly higher rates of Clavien-Dindo Grade $\geq 3$ complications $(p=0.001)$, all cause 30 -day mortality ( $8.5 \%$ vs. $2.9 \%, p=0.028$ ), but no significant difference was observed in operative 30-day mortality no modification [19]. The authors suggested that the increase in the number of complications would be more related to the change in the emergency population during COVID-19 epidemic (older patients with multiple co-morbidities), a change already noted in the literature for other surgical specialties $[20,21]$. This also might be in line with the higher proportion of patients with obesity during the lockdown in our data.

Another hypothesis was that the use of methotrexate as conservative treatment of ectopic pregnancy could have been increased to avoid hospitalizations. This could have increased the frequency of ruptured ampullas, but it turned out that methotrexate was used normally during this period.

Although this work presents one of the largest series of gynecological emergencies during the lockdown, it is important to highlight the limitations of our results. First, our study was retrospective in nature and a few features would have been very valuable to analyze (for example, time spent in emergency room could not be retrieved). However, there were no missing values on any of the collected variables.

In addition, some non-significant results could be due to the low statistical power: vital emergencies were rare phenomena given numerous events of moderate seriousness/ severity seriousness. Still, this is a true reflection of the population in gynecological emergencies.

Finally, the overall period of the study was quite short. This study did not focus on the post-confinement period. Thus, future additional long-term studies to evaluate the potential impact of the lockdown will be needed. Indeed, this series is only a preliminary work whose purpose is to provide food for thought in order to organize our management.

Despite these few limitations, our study documented a strong effect of lockdown on the number of admissions to gynecological EDs. An important strength of this work is the consideration of the period effect and not just a "before and after" effect.

\section{Conclusions}

The COVID-19 epidemic was a test for the capacity of health systems to manage emergencies and to undergo a paradigm shift. The increase in the number of emergency hospitalizations and surgeries despite a significant decrease in consultations during the lockdown confirms the need for 
hospitals to be constantly resilient and prepared to a hypothetic sanitary disaster.

\section{Conflict of interest}

The authors declare no conflict of interest.

\section{References}

1. panorama de la DREES. Healthcare institutions - 2020 edition - Ministère des Solidarités et de la Santé [Internet]. [cité 26 sept 2020]. Disponible sur: https://drees.solidarites-sante.gouv.fr/ etudes-et-statistiques/publications/panoramas-de-la-drees/article/ les-etablissements-de-sante-edition-2020.

2. cour des comptes.social_security_report_2014_hospital_emergencies.pdf [Internet]. [cité 26 sept 2020]. Disponible sur: https:// www.ccomptes.fr/sites/default/files/EzPublish/rapport_securite_ sociale_2014_urgences_hospitalieres.pdf

3. Curtis KM, Hillis SD, Kieke BA, et al. Visits to emergency departments for gynecologic disorders in the United States, 1992-1994. Obstet Gynecol 1998; 91: 1007-12.

4. Nicholson WK, Ellison SA, Grason H, Powe NR. Patterns of ambulatory care use for gynecologic conditions: a national study. Am J Obstet Gynecol 2001; 184: 523-30.

5. Alouini S, Mesnard L, Coly S, et al. Gynecological emergencies: etiology and degree of gravity. J Gynecol Obstet Biol Reprod 2012; 41: 48-54.

6. Stoecklin SB, Rolland P, Silue Y, et al. First cases of coronavirus disease 2019 (COVID-19) in France: surveillance, investigations and control measures, January 2020. Euro Surveill Bull Eur Sur Mal Transm Eur Commun Dis Bull 2020; 25: 2000094.

7. Kayem G, Lecarpentier E, Deruelle P, et al. A snapshot of the Covid-19 pandemic among pregnant women in France. J Gynecol Obstet Hum Reprod 2020; 49: 101826.

8. Maringe C, Spicer J, Morris M, et al. The impact of the COVID-19 pandemic on cancer deaths due to delays in diagnosis in England, UK: a national, population-based, modelling study. Lancet Oncol 2020; 21: 1023-34

9. Rahul, Verma A, Yadav P, et al. Non-COVID surgical emergency during the nationwide lockdown due to corona pandemic: a critical appraisal. Indian J Surg 2020; 1-5. doi: 10.1007/s12262-02002549-5.

10. Grandi G, Savio MCD, Caroli M, et al. The impact of COVID-19 lockdown on admission to gynecological emergency departments: results from a multicenter Italian study. Int J Gynecol Obstet 2020. doi: 10.1002/ijgo.13289.

11. Isba R, Edge R, Auerbach M, et al. COVID-19. Pediatr Emerg Care 2020. doi: 10.1097/PEC.0000000000002260.

12. Ojetti V, Covino M, Brigida $M$, et al. Non-COVID diseases during the pandemic: where have all other emergencies gone? Medicina 2020; 56: 512.

13. Wickham H, Averick M, Bryan J, et al. Welcome to the tidyverse. J Open Source Softw 2019; 4: 1686.

14. R Core Team. R: A Language and Environment for Statistical Computing [Internet]. Vienna, Austria: R Foundation for Statistical Computing; 2020. Disponible sur: https://www.R-project.org/

15. Garrafa E, Levaggi R, Miniaci R, Paolillo C. When fear backfires: emergency department accesses during the Covid-19 pandemic. Health Policy 2020; 124: 1333-9.
16. Oikonomou E, Aznaouridis K, Barbetseas J, et al. Hospital attendance and admission trends for cardiac diseases during the COVID-19 outbreak and lockdown in Greece. Public Health 2020; 187: 115-9.

17. Kilfoyle KA, Vrees R, Raker CA, Matteson KA. Non-urgent and urgent emergency department use during pregnancy: an observational study. Am J Obstet Gynecol 2017; 216: 181.e1-7.

18. Uscher-Pines L, Pines J, Kellermann A, et al. Deciding to visit the emergency department for non-urgent conditions: a systematic review of the literature. Am J Manag Care 2013; 19: 47-59.

19. McLean RC, Young J, Musbahi A, et al. A single-centre observational cohort study to evaluate volume and severity of emergency general surgery admissions during the COVID-19 pandemic: Is there a "lockdown" effect? Int J Surg 2020; 83: 259-66.

20. Cano-Valderrama O, Morales X, Ferrigni CJ, et al. Reduction in emergency surgery activity during COVID-19 pandemic in three Spanish hospitals. Br J Surg 2020; 107: e239.

21. Novara G, Bartoletti R, Crestani A, et al. Impact of the COVID-19 pandemic on urological practice in emergency departments in Italy. BJU Int 2020; 126: 245-7. 